\title{
Adaptive Tangential Relaxation and Surface Fitting for High-Order Mesh Optimization
}

\author{
Veselin A. Dobrev ${ }^{\dagger}$, Patrick Knupp ${ }^{\ddagger}$ Tzanio V. Kolev ${ }^{\dagger}$, \\ Ketan Mittal ${ }^{\dagger}$ and Vladimir Z. Tomov* ${ }^{* \dagger}$ \\ $\dagger$ Lawrence Livermore National Laboratory \\ 7000 East Avenue, Livermore, CA 94550, United States \\ e-mails: dobrev1@llnl.gov, kolev1@llnl.gov, mittal3@llnl.gov, tomov2@llnl.gov \\ $\ddagger$ Dihedral LLC \\ 37 Zane Grey Way Bozeman, MT, 59715, United States \\ e-mail: knupp.patrick@gmail.com
}

\begin{abstract}
We propose a new approach for controlling the characteristics of certain mesh faces during optimization of high-order curved meshes. The practical goals are tangential relaxation along initially aligned curved boundaries and internal surfaces, and mesh fitting to initially non-aligned surfaces. The distinct feature of the method is that it utilizes discrete finite element functions (for example level set functions) to define implicit surfaces, which are used to adapt the positions of certain mesh nodes. The algorithm does not require CAD descriptions or analytic parametrizations, and can be beneficial in computations with dynamically changing geometry, for example shape optimization and moving mesh multi-material simulations.

The problem is formulated as a variational minimization of a chosen mesh-quality metric, through the Target-Matrix Optimization Paradigm [1], with additional penalty terms that enforce the desired tangential motion and/or surface alignment. These penalty terms connect the concept of mesh motion to the discrete finite element function that defines the desired node position. As the penalty terms depend on discrete functions, there is an interpolation procedure that makes the functions available on different meshes [2]. The optimization method is based on global node movement and does not alter the topology of the starting mesh.

The main advantage of this approach is that it completely avoids geometrical operations (for example surface projections), and all calculations can be performed through finite element operations. Thus the main steps of the method are independent of dimension, order of the mesh, and types of elements. The drawback is that the notions of alignment and fitting are always approximate (imposed weakly), so that the method may not be the best choice in situations that require the exact preservation of certain features, e.g., for representation of sharp edges in 3D.
\end{abstract}

\section{REFERENCES}

[1] Veselin A. Dobrev and Patrick Knupp and Tzanio V. Kolev and Ketan Mittal and Vladimir Z. Tomov. The Target-Matrix Optimization Paradigm for High-Order Meshes. SIAM J. Sci. Comp., Vol. 41(1), pp. B50-B68, (2019). pp. 285-302, (2019).

[2] Veselin A. Dobrev, Patrick Knupp, Tzanio V. Kolev and Vladimir Z. Tomov. Towards Simulation-Driven Optimization of High-Order Meshes by the Target-Matrix Optimization Paradigm. 27th International Meshing Roundtable, pp. 285-302, (2019).

Performed under the auspices of the U.S. Department of Energy under Contract DE-AC52-07NA27344 (LLNL-ABS-818320) 